\title{
Quantum metrology to probe atomic parity nonconservation
}

\author{
P. Mandal* and M. Mukherjee \\ Raman Center for Atomic Molecular and Optical Sciences, \\ Indian Association for the Cultivation of Science, Kolkata -700 032
}

(Dated: Received November 1, 2018)

\begin{abstract}
An entangled state prepared in a decoherence free sub-space together with a Ramsey type measurement can probe parity violation in heavy alkali ions like $\mathrm{Ba}^{+}$or $\mathrm{Ra}^{+}$. Here we propose an experiment with $\mathrm{Ba}^{+}$as an example to measure the small parity violating effect in this system. It has been shown that a measurement on a maximally correlated system will reduce the uncertainty as compared to that on a single ion measurement. In addition it also provides a feasible solution to measure the nuclear spin dependent part of the total parity violating light shift in an ionic system which has so far not been addressed.
\end{abstract}

PACS numbers: 03.65.Ta, 42.50.Lc 32.80.-t, 42.50.Ct, 42.50.Vk, 32.80.Lg

Measurement of atomic parity nonconservation (PNC) in the $6 S-7 S$ transition of atomic Cs has been performed with an uncertainty reaching $0.35 \%[1-3]$. An equally demanding theoretical effort in this atom [4] leads to the evaluation of the weak nuclear charge $Q_{W}$ which is a unique low energy test of the standard electroweak theory. Further improvement in the precision will lead to reducing the limits on the mass of an eventually additional light or heavy boson [5]. Apart from the necessity of improving the PNC measurement in $\mathrm{Cs}$, it would be worthwhile to consider other possible experimental techniques for the measurement of PNC in other systems. Recently the largest PNC effect has been measured in the $6 s^{2}{ }^{1} S_{0}-5 d 6 s^{3} D_{1}$ transition in atomic yetterbium [6] employing the same technique as used in the Cs experiment. The enhancement in this case is caused by degeneracy of atomic levels 7]. Though the measured PNC dipole transition amplitude $\left(E 1_{P N C}\right)$ is 100 times larger compared to that in atomic cesium, the experimental precision is not good enough to verify the Standard Model or to predict any Physics beyond it.

A newly proposed method adopted for the Cs measurement involved left-right asymmetry of the forbidden transition rate in $6 S-7 S$ transition [8, 9]. This method is presently been pursued for Fr, the heaviest alkali [10, 11]. Unfortunately, the requirement of a large number of atoms to observe the asymmetry limits this experiment. Recently, it has been proposed to observe a linear Stark shift in an interferometric measurement with small number of atoms of Fr [12]. The measurement of light shift arising due to the interference between $E 1_{P N C}$ and electric quadrupole transition amplitude $E 2$ in a heavy ion like $\mathrm{Ba}^{+}, \mathrm{Ra}^{+}$proposed by Fortson 13] seems to be the most promising technique. It can, in principle achieve a precision of $0.1 \%$. Presently it is being pursued at different experimental laboratories [14, 15]. Initial radio frequency $(\mathrm{RF})$ spectroscopy on $\mathrm{Ba}^{+}$has also been performed to observe the light shifts

\footnotetext{
*drupm@iacs.res.in
}

of different Zeeman sublevels. The major limitations appear in these measurements are from the magnetic field noise as well as laser frequency noise [16]. In order to finally observe the PNC induced light shift it is necessary to achieve an uncertainty well below one Hertz in the ground state Larmor frequency since even in the presence of a strong electric field, the shift is only of the order of $0.2 \mathrm{~Hz}$. Though, maximally entangled states for quantum metrology is a rather recent field, it has already been implemented in a relatively few cases [17 19]. They have been used to improve the signal-to-noise ratio [20], to efficiently detect quantum state [21], to measure scattering length 22] and to do spectroscopy in decoherence free sub-space (DFS) [23]. An entangled state prepared in a DFS [24, 25] makes any measurement immune to environmental changes. Thereby, this can be effectively used to overcome the magnetic field noise limitation of the single ion experiment to observe the PNC light shift. In what follows, we outline this promising technique of such a measurement with high precision.

Parity nonconservation in an atomic system leads to a small mixing between states of opposite parities resulting a nonzero probability in the electric dipole transition which is strictly forbidden by parity conservation rule. The effect, though scales as $Z^{3}$ for heavier atoms [3], is on the order of $10^{-11} e a_{0}$. It is thus an experimental challenge to measure such a small quantity directly. Instead, people look for an interference-like phenomena between $E 1_{P N C}$ and a much stronger higher order electromagnetic transition between the same states. For $\mathrm{Ba}^{+}$ or $\mathrm{Ra}^{+}$such an interference between $E 1_{P N C}$ and $E 2$ in $\mathrm{nS}_{1 / 2}-(\mathrm{n}-1) \mathrm{D}_{3 / 2}$ transition is proposed to measure the vector light shift [13]. In presence of the electric field of a laser

$$
\mathbf{E}(\mathbf{r}, t)=\frac{1}{2} \mathbf{E}_{0}\left[e^{i(\mathbf{k} . \mathbf{r}-\omega t)}+\text { c.c. }\right],
$$

the $E 1_{P N C}$ and $E 2$ couplings between $\mathrm{S}_{1 / 2}-\mathrm{D}_{3 / 2}$ are described in terms of respective Rabi frequencies as

$$
\Omega_{m^{\prime} m}^{P N C}=\frac{1}{2 \hbar} \sum_{i} \varepsilon_{m^{\prime} m}^{P N C} E_{i}(0)
$$




$$
\Omega_{m^{\prime} m}^{Q}=\frac{1}{2 \hbar} \sum_{i, j} \varepsilon_{m^{\prime} m}^{Q}\left[\frac{\partial E_{i}(r)}{\partial x_{j}}\right]_{0}
$$

$r=0$ being the position of the ion in the trap. Here $\varepsilon_{m^{\prime} m}^{P N C}$ and $\varepsilon_{m^{\prime} m}^{Q}$ describe $E 1_{P N C}$ and $E 2$ matrix elements between $m$ sublevel of $\mathrm{S}_{1 / 2}$ and $m^{\prime}$ sublevel of $\mathrm{D}_{3 / 2}$. The resultant Rabi frequency of $m$ sublevel of $\mathrm{S}_{1 / 2}$ is [13]

$$
\Omega_{m} \approx \Omega_{m}^{Q}+\operatorname{Re} \sum_{m^{\prime}}\left(\Omega_{m^{\prime} m}^{P N C *} \Omega_{m^{\prime} m}^{Q}\right) / \Omega_{m}^{Q},
$$

where $\left(\Omega_{m}\right)^{2}=\sum_{m^{\prime}}\left|\Omega_{m^{\prime} m}\right|^{2}=\sum_{m^{\prime}}\left|\Omega_{m^{\prime} m}^{Q}+\Omega_{m^{\prime} m}^{P N C}\right|^{2}$ and $\left(\Omega_{m}^{Q}\right)^{2}=\sum_{m^{\prime}}\left|\Omega_{m^{\prime} m}^{Q}\right|^{2}$. Considering the Zeeman splitting of the magnetic sublevels to be comparable to the line width of $\mathrm{S}_{1 / 2}-\mathrm{D}_{3 / 2}$ laser, the light shift of $m$ sublevel of the ground state is given by

$$
\Delta \omega_{m}=\delta / 2-\Omega_{m}
$$

where $\delta=\omega_{0}-\omega$ is the detuning of the laser frequency from the atomic transition frequency. It is convenient to drive both the quadrupole and PNC allowed $\mathrm{S}_{1 / 2}-\mathrm{D}_{3 / 2}$ dipole transition independently so that much larger contribution in $\Delta \omega_{m}$ due to the pure $E 2$ coupling remains same while that due to the interference term changes sign for the magnetic sublevels of ground state. Fortson showed 13 that it is achieved when a single ion is placed $(x=z=0)$ simultaneously at the antinode and the node of two standing wave lasers represented respectively as

$$
E^{\prime}=\hat{x} E_{0}^{\prime} \cos k z, E^{\prime \prime}=i \hat{z} E_{0}^{\prime \prime} \sin k x .
$$

These lasers produce $\Delta m= \pm 1$ dipole and quadrupole transitions respectively. In presence of these two lasers the Larmor frequency between the ground magnetic sublevels is given by

$$
\omega_{L}^{\prime}=\omega_{L} \sim 2 \operatorname{Re} \sum_{m^{\prime}}\left(\Omega_{m^{\prime} m}^{P N C *} \Omega_{m^{\prime} m}^{Q}\right) / \Omega_{m}^{Q},
$$

where $\omega_{L}$ is the Larmor frequency between the same sublevels in absence of the lasers. Thus the PNC shift can be extracted from the measurement of the ground state Larmor frequency in absence and in presence of the laser fields. Fortson calculated the shift to be $0.2 \mathrm{~Hz}$ for $\mathrm{Ba}^{+}$ in presence of strong laser field $E_{0}^{\prime}=2 \times 10^{6} \mathrm{~V} / \mathrm{m}[13$ ]. However, it is still a challenge to measure such a small change by applying usual RF spectroscopic technique. It demands a magnetic field of stability one part in $10^{8}$ for few hundreds of a $\mathrm{kHz}$ magnetic splitting in order to achieve an accuracy $1 \%$.

Employing the generalized Ramsey interferometric technique to maximally correlated atomic state it is possible to determine the PNC light shift with the required precision. Under free precision a maximally entangled atomic state, like one of the Bell's states, $\psi(0)=$ $\frac{1}{\sqrt{2}}\left(\left|u_{1}\right\rangle\left|u_{2}\right\rangle+\left|v_{1}\right\rangle\left|v_{2}\right\rangle\right)$ evolves into $\psi(\tau)=\frac{1}{\sqrt{2}}\left(\left|u_{1}\right\rangle\left|u_{2}\right\rangle+\right.$ $\left.\exp ^{i \Delta \lambda \tau}\left|v_{1}\right\rangle\left|v_{2}\right\rangle\right)$ after a time $\tau$. The phase evolution rate $\Delta \lambda=\left[\left(E_{u_{1}}+E_{u_{2}}\right)-\left(E_{v_{1}}+E_{v_{2}}\right)\right] / \hbar$ corresponds to the

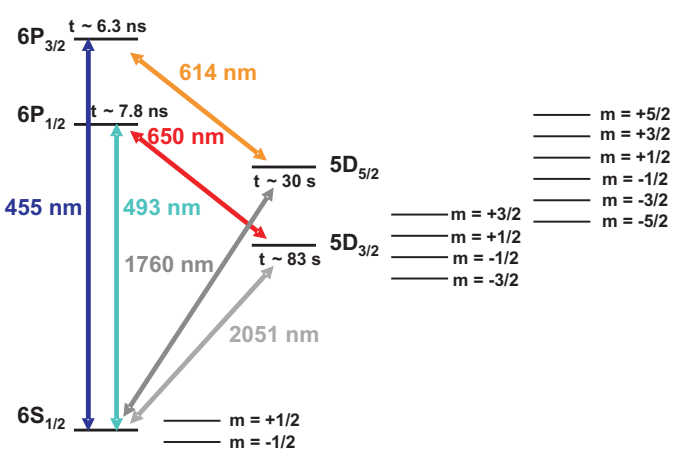

FIG. 1: (Color online) Relevant atomic levels of $\mathrm{Ba}^{+}$. The Zeeman sublevels are also shown for clarity.

energy difference between the atomic states $u_{k}$ and $v_{k}$. The real part of the phase factor $\exp ^{i \Delta \lambda t}$ can be measured by projecting the ions on the states $| \pm\rangle=\frac{1}{\sqrt{2}}\left(|u\rangle_{k} \pm|v\rangle_{k}\right)$ and measuring the relative phase. For states in the DFS the free precision time $\tau$ can be made very long and hence the phase can be measured accurately [25, 26]. By a careful choice of the state it is possible to measure the PNC shift in DFS thereby avoiding the possible systematic effects in coupling to the environment.

Instead of a single ion, in the following we consider a string of two $\mathrm{Ba}^{+}$ions (even isotope, $I=0$ ) confined in a linear Paul trap. The relevant electronic levels are shown in fig. 11 The ions cooled into their ground motional state of the first two normal modes of motion 27] using laser Doppler cooling applying $493 \mathrm{~nm}$ and $650 \mathrm{~nm}$ lasers followed by sideband cooling with $1.76 \mu \mathrm{m}$ laser [fig. 1. Both the ions are prepared in a Zeeman sublevel of the ground electronic state (say $6 S_{1 / 2}, m=1 / 2$ for example). The ions are then individually treated with a sequence of laser pulses. A $\pi / 2$ pulse at the blue sideband on the first ion prepares it in a superposition of electronic ground and metastable (say $D_{5 / 2}, m=1 / 2$ say for example) state and motional ground and first excited state. A $\pi$ pulse at the carrier on the second ion brings it to electronic excited state $\left(D_{5 / 2}, m=1 / 2\right)$ keeping the motional state unchanged. Another blue sideband $\pi$ pulse on the second ion transfers the excited state population back to ground electronic and motional states. One more $\pi$ pulse at the carrier on each ion coherently transfers the quadrupole excited state population into other Zeeman sublevel in the ground state thus preparing the state

$$
|\Psi\rangle=\frac{1}{\sqrt{2}}\left(|1\rangle_{1}|0\rangle_{2}+|0\rangle_{1}|1\rangle_{2}\right)|0\rangle,
$$

where $|1\rangle_{i}$ and $|0\rangle_{i}$ stand for $m=1 / 2$ and $-1 / 2$ of $6 \mathrm{~S}_{1 / 2}$ state of $i^{\text {th }}$ ion and $|0\rangle$ describes the ground motional state of center-of-mass (COM) mode. The presence of the two ion state makes it decoherence free as compared to the superposition state of a single ion. The Zeeman shifts of the two parts of the entangled state cancel out in absence of magnetic field gradient along the trap axis. This state is immune to any decoherence effects arising 


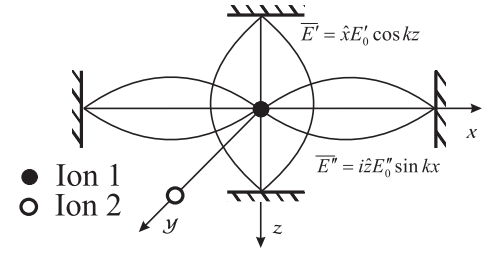

FIG. 2: A schematic of the experiment with two ions placed in a linear ion trap and interrogated by two standing wave lasers. The amplitude $E_{0}^{\prime}$ should be orders of magnitude larger as compared to $E_{0}^{\prime \prime}$ for improved systematic.

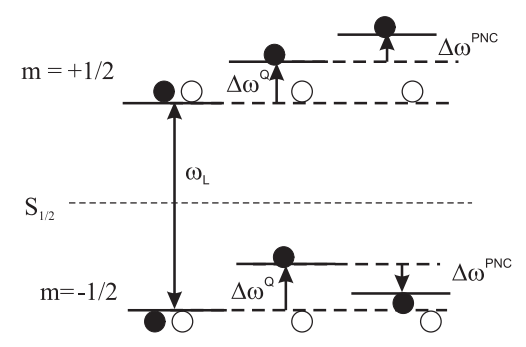

FIG. 3: A cartoon of energy shifts of ground state magnetic sublevels of two ions in presence of the magnetic field and lasers $E^{\prime}, E^{\prime \prime} . \Delta \omega^{Q}$ and $\Delta \omega^{P N C}$ denotes quadrupole and PNC light shifts respectively.

from the magnetic field fluctuation common to both ions, spontaneous decay etc and therefore the state, in principal possesses an infinitely long coherence.

After preparing such an entangled state in DFS, two laser fields $E^{\prime}$ and $E^{\prime \prime}$ in a standing wave configuration are applied for a time interval $\tau$ on one ion (Ion 1, say) as shown in fig. 2. The magnetic splitting, quadrupole light shift and PNC light shift of the ground state magnetic sublevels for the two ions are shown schematically in fig. 3. It depicts that the ground state Larmor frequency of one ion shifts only due to PNC interaction while that remains unchanged for the other ion. Thus a small perturbation is introduced within the entangled state $|\Psi\rangle$ (Eq. 8) and it evolves as

$$
|\Psi(\tau)\rangle=\frac{1}{\sqrt{2}}\left(|1\rangle_{1}|0\rangle_{2}+\exp (i \Delta \lambda \tau)|0\rangle_{1}|1\rangle_{2}\right)|0\rangle,
$$

where the phase evolution rate $\Delta \lambda$ corresponds to the energy difference between the two parts of the entangled state i.e. the PNC light shift given by Eq. 7

The state $|\Psi(\tau)\rangle$ should be projected on $| \pm\rangle=$ $\frac{1}{\sqrt{2}}\left(|0\rangle_{i} \pm|1\rangle_{i}\right)$ in order to observe the time evolution of the expectation value $\left\langle\sigma_{x}^{(1)} \otimes \sigma_{x}^{(2)}\right\rangle$, where $\sigma_{x}^{(i)}$ denotes the Pauli spin matrix for $i^{t h}$ ion. It oscillates with a frequency $2 \pi / \Delta \lambda[25]$. Thus the PNC light shift can directly be extracted from the measurement of the oscillation frequency.

The uncertainty in the PNC light shift measurement will be determined by the decoherence time of the maximally entangled state which is practically infinite in absence of external perturbation but limited by the natural life time $(\tau)$ of $5 D_{3 / 2}$ state in our case. The uncertainty in the frequency measurement on $N$ maximally correlated atomic systems is inversely proportional to $N T$ instead of $\sqrt{N} T$ for uncorrelated systems 17. Here $T$ is the time of a single measurement which can be made as large as $\tau$ is. Therefore the statistical signal-to-noise ratio for $n$ no. of measurements can be approximated as

$$
\frac{\varepsilon^{\mathrm{PNC}}}{\delta \varepsilon^{\mathrm{PNC}}} \approx \frac{\varepsilon^{\mathrm{PNC}} E_{0}^{\prime}}{\hbar} f \sqrt{n} N \tau,
$$

where $f$ signifies an experimental efficiency factor. It is determined by how well the entangled state is formed and detected. In our case it can be close to one since it has been shown that such state can be prepared with a fidelity of nearly $95 \%$. $N$ in this case is 2 since two ion maximally correlated state is used for the measurement. Considering same $f$ as in single ion experiment [13], the figure-of-merit will be 2 times higher in present experiment. In other words, it would be possible to achieve the same precision by performing $1 / 4^{t h}$ no. of experiments as compared to that on single ion. It can in principle be further improved by considering the correlated state of more than two ions.

The size of the PNC light shift could in principle be increased by increasing the amplitude $E_{0}^{\prime}$. However, as the amplitude is increased, the off-resonant couplings become more and more important which effectively deteriorate the coherence of the entangled state. The induced loss rate is 28]

$$
\Gamma_{\gamma j m}^{\text {loss }}=\frac{e^{2}}{4 \hbar^{2}} \sum_{\gamma^{\prime}, m^{\prime}, \pm \omega} \frac{\left|\left\langle\gamma^{\prime} j^{\prime} m^{\prime}|\mathbf{E} \cdot \mathbf{r}| \gamma j m\right\rangle\right|^{2}}{\left(\omega_{\gamma^{\prime}}-\omega_{\gamma} \pm \omega\right)^{2}} \frac{\omega^{3}}{\left(\omega_{\gamma^{\prime}}-\omega_{\gamma}\right)^{3}} \Gamma_{\gamma^{\prime} j^{\prime}},
$$

where $\Gamma_{\gamma^{\prime} j^{\prime}}$ is the spontaneous transition rate out of $\left|\gamma^{\prime} j^{\prime}\right\rangle$. Considering the off-resonant coupling from $6 \mathrm{P}$ levels $\Gamma_{\gamma j m}^{\text {loss }}$ for the states $\left|6 S_{1 / 2}, m=1 / 2\right\rangle, \mid 5 D_{3 / 2}, m=$ $1 / 2\rangle$ and $\left|5 D_{3 / 2}, m=3 / 2\right\rangle$ of $\mathrm{Ba}^{+}$has been estimated for an electric field $E_{0}^{\prime}=1.6 \times 10^{6} \mathrm{~V} / \mathrm{m}$ and it turns out to be $0.0044,0.007$ and $0.0008 \mathrm{~Hz}$ respectively. The total induced loss rate is comparable to the natural decay rate of $5 \mathrm{D}_{3 / 2}(0.012 \mathrm{~Hz})$ and hence the electric field amplitude mentioned earlier is the maximum for this experiment. The off-resonant light shift for such a larger electric field is also significant, but for a linear polarization the ground state Zeeman sublevels suffer scalar shift and it does not change the Larmor frequency. However, in presence of small circular polarization in $E^{\prime}$ laser the sublevels experience a vector shift [29] that can mimic PNC measurement. This systematic can be measured by performing the same experiment described above but in absence of $E^{\prime \prime}$ laser so that there is no interference. Alternatively, the ions could be placed at the antinodes of $E^{\prime}$ laser while one of them at the node of $E^{\prime \prime}$ laser. A small magnetic field gradient along the trap axis is a major source of systematic but it can also be eliminated by repeating the experiment by exchanging the role of the two ions or on the same ion in absence of the laser fields. In 
order to finally extract the PNC induced $E 1$ amplitude it is necessary to know the electric field at the ion position $E_{0}^{\prime}(0), E_{0}^{\prime \prime}(0)$ and the quadrupole light shift. The electric fields could be measured by off-resonant excitations since the related matrix elements for $\mathrm{Ba}^{+}$are well known [30]. The quadrupole light shift can as well be measured by using the technique of generalized Ramsey interference experiment [31, 32]. Using two ions instead of one ion in a linear ion trap may lead to unwanted stray electric field which is a major concern for parity mixing. Since the ions are side band cooled to the ground state of their COM mode, the field at the ion equilibrium position must be zero. The ions in a linear string of Coulomb crystal have a wavepacket span which is negligible as compared to the wavelength of the standing wave. Therefore they can be considered to be at rest. The first order effects due to stray fields as well as the trapping potential are not only displaced from the PNC transition by multiples of trap frequency but are also negligibly small due to sideband cooling.

In case of non-zero nuclear spin isotopes the Physics of $\mathrm{PNC}$ is even richer because of the presence of a tiny nuclear spin dependent contribution. The measurement of the nuclear spin dependent (NSD) part and hence the nuclear anapole moment in $E 1_{P N C}$ appears to be difficult by driving RF spin flip transition on a single ion but it is feasible with the technique described here. For example, in spin $I=3 / 2$ isotopes there is only one $M 1$ allowed transition (between $m_{F}=1,0$ of $F=2, S_{1 / 2}$ in presence of laser fields connecting $F=2, S_{1 / 2}$ and $F^{\prime}=3, D_{3 / 2}$ ) where the quadrupole transition induced light shift does not change the Larmor frequency. This is essential for measuring the total PNC light shift. In order to extract the NSD part in $E 1_{P N C}$, other transitions of the same isotope need to be considered to measure the light shift due to the total PNC amplitude. The Larmor frequency between those two levels contains not only the differential PNC shift but also the differential quadrupole light shift which is a serious systematic effect. However, two entangled states (Eq. 8) with $|1\rangle_{i}=\left|m_{F}=2, F=2,6 S_{1 / 2}\right\rangle_{i}$ , $|0\rangle_{i}=\left|m_{F}=-2, F=2,6 S_{1 / 2}\right\rangle_{i}$ and $|1\rangle_{i}=\mid m_{F}=$ $\left.1, F=2,6 S_{1 / 2}\right\rangle_{i},|0\rangle_{i}=\left|m_{F}=-1, F=2,6 S_{1 / 2}\right\rangle_{i}$ can be formed to measure the NSD contribution. The measured light shifts with these two states contain both NSD and NSI parts which are same in two transitions but multiplied by associated Clebsch-Gordan coefficients. It is therefore, convenient to separate out both contributions with high precision.

We have shown that a two ion entangled state is a better tool for the measurement of parity violating light shift as compared to the single ion experiment. Various systematics present in a single ion experiment are absent in this case and some of them can even be measured in this case. The statistical signal-to-noise ratio can be improved with this maximally correlated state. The measurement of nuclear spin dependent contribution and nuclear anapole moment is feasible using correlated atomic states as shown here. The experimental techniques involved here are regularly in use by the quantum computation community. Therefore it is feasible with today's technology.

\section{Acknowledgements.}

The authors would like to thank Prof. B. P. Das at IIA, Bangalore and Dr. C. F. Roos at IQOQI, Innsbruck for fruitful discussions. P. Mandal acknowledges the financial support from CSIR, India. We acknowledge the financial support of SERC, DST.
[1] C. S. Wood et al., Science 275, 1759 (1997).

[2] S. C. Bennett, C. E. Wieman, Phys. Rev. Lett. 82, 2484 (1999).

[3] M. A. Bouchiat et al., Rep. Prog. Phys. 60, 1351 (1997).

[4] J. S. M. Ginges et al., Phys. Rep. 397, 63 (2004) and referneces therein.

[5] C. Bouchiat et al., Phys. Lett. B608, 87 (2005); R. D. Young, R. D. Carlini, A. W. Thomas, J. Roche, Phys. Rev. Lett. 99, 122003 (2007).

[6] K. Tsigutkin et al., Phys. Rev. Lett. 103,071601 (2009).

[7] D. DeMille, Phys. Rev. Lett. 74,4165 (1995).

[8] J. Guéna et al., Phys. Rev. Lett. 90, 143001 (2003).

[9] J. Guéna et al., J. Opt. Soc. Am. B22, 21 (2005).

[10] E. Gomez et al., Rep. Prog. Phys. 69, 79 (2006).

[11] S. Aubin et al., Rev. Sci. Instrum. 74, 4342 (2003).

[12] M. A. Bouchiat, Phys. Rev. Lett. 100, 123003 (2008).

[13] N. Fortson, Phys. Rev. Lett. 70, 2383 (1993).

[14] O. O. Versolato et al., Phys. Rev. A82, 010501(R) (2010).

[15] P. Mandal et al., Hyperfine Interact. 196, 261 (2010).

[16] J. Sherman, T. W. Koerber, A. Markhotok, W. Nagourney, E. N. Fortson, Phys. Rev. Lett. 94, 243001 (2005).

[17] J. J. Bollinger, W. M. Itano, D. J. Wineland,
D. J. Heinzen, Phys. Rev. A54, R4649 (1996).

[18] V. Giovannetti, S. Lloyd, L. Maccone, Science 306, 1330 (2004).

[19] V. Giovannetti et al., Phys. Rev. Lett. 96, 010401 (2006).

[20] D. Leibfried et al., Science 304, 1476 (2004).

[21] P. O. Schimdt et al., Science 309, 749 (2005).

[22] A. Widera et al., Phys. Rev. Lett. 92, 160406 (2004).

[23] C. F. Roos et al., Nature 443, 05101 (2006).

[24] D. A. Lidar, I. L. Chuang, K. B. Whaley, Phys. Rev. Lett 81, 2594 (1998).

[25] C. F. Roos et al., Phys. Rev. Lett. 92, 220402 (2004).

[26] H. Häffner et al., Appl. Phys. B81, 151 (2005).

[27] Ch. Roos et al., Phys. Rev. Lett. 83, 4713 (1999).

[28] T. W. Koerber, PhD thesis, University of Washington (2003).

[29] J. E. Stalnaker et al., Phys. Rev. A73, 043416 (2006).

[30] B. K. Sahoo, L. W. Wansbeek, K. Jungmann, R. G. E. Timmermans, Phys. Rev. A79, 052512 (2009).

[31] H. Häffner et al., Phys. Rev. Lett. 90, 143602 (2003).

[32] N. F. Ramsey, Molecular Beams (Oxford University, New York, 1985). 